\title{
Factores cognitivos relacionados con la capacidad de cálculo de división en estudiantes de 4ํaño de educación primaria en Argentina
}

Cognitive factor related to division ability in fourth grade students of primary education in Argentina

\section{Volumen 18, Número 1}

Enero-Abril

pp. 1-26

\author{
Florencia Stelzer \\ Isabel Introzzi \\ María Laura Andrés \\ María Marta Richard`s \\ Sebastián Urquijo
}

Revista indizada en REDALYC, SCIELO

Revista distribuida en las bases de datos:

LATINDEX, DOAJ, REDIB, IRESIE, CLASE, DIALNET, SHERPA/ROMEO, QUALIS-CAPES, MIAR

Revista registrada en los directorios:

ULRICH'S, REDIE, RINACE, OEI, MAESTROTECA, PREAL, $\underline{\text { CLACSO }}$ 


\title{
Factores cognitivos relacionados con la capacidad de cálculo de división en estudiantes de $4^{\circ}$ año de educación primaria en Argentina
}

Cognitive factor related to division ability in fourth grade students of primary education in Argentina

\author{
Florencia Stelzer ${ }^{1}$ \\ Isabel Introzzi2 \\ María Laura Andrés ${ }^{3}$ \\ María Marta Richard 's ${ }^{4}$ \\ Sebastián Urquijo ${ }^{5}$
}

\begin{abstract}
Resumen: Diferentes estudios indicaron que el aprendizaje de la matemática se asocia con los recursos cognitivos que dispone el estudiantado para afrontar su construcción. Sin embargo, la relación específica de procesos y habilidades cognitivas con la capacidad de cálculo de operaciones de división ha sido escasamente analizada. El objetivo de este artículo empírico es analizar la relación de la capacidad de cálculo de operaciones de división con el rendimiento en memoria de trabajo, inhibición e inteligencia fluida. La muestra fue seleccionada por disponibilidad y estuvo integrada por 135 estudiantes de 4 año del sistema educativo argentino (participantes de 9 y 10 años de edad). Los mismos fueron evaluados dentro de los establecimientos educativos a lo que asistían, durante el horario habitual de clases, a través de diferentes tareas que permitían la medición de tales variables. Los resultados indicaron que los estudiantes con distinto nivel de desempeño en división diferían entre sí en todas las variables cognitivas estudiadas (a excepción de la inhibición). Este hallazgo muestra que la inteligencia fluida y memoria de trabajo se relacionan con el conocimiento de la división en estudiantes de 4ำ año de la escolaridad primaria. Asimismo, sugiere que el aprendizaje de esta operación podría facilitarse al considerar las diferencias individuales en estos factores cognitivos durante el diseño y selección de los materiales didácticos y estrategias pedagógicas a utilizar para su enseñanza.
\end{abstract}

Palabras clave: educación primaria, análisis matemático, cognición

Abstract: Different studies have shown a relationship between mathematics learning and cognitive factors; however, the specific link between division and cognitive abilities has been less studied. Previous research has shown that division ability during elementary school years is one of the most important predictors' factors of general mathematic achievement in high school. Therefore, recognizing the cognitive factors related to division is important to improve mathematic learning in high school. The aim of this study was analyzed the relationship among division, working memory, inhibition and fluid intelligence. The sample was choose by the availability of the participants and it was integrated by 135 4th grade students (ages: 9-10) from the Argentine educational system. They were assessed inside their school during regular classes schedule using different task. The results indicate that the students with different level in the division achievement diverge between them in the cognitive variables studied (inhibition was an exception). This conclusion showed that students' differences in division achievement were related to working memory and fluid intelligence. Those findings show a relation between intelligence and working memory and suggest that division learning could be improved by considering individual differences on those cognitive factors.

Keywords: primary education, mathematical analysis, cognition

${ }^{1}$ Investigadora Asistente del Concejo Nacional de Investigaciones Científicas y Técnicas (CONICET), Argentina. Doctora en Psicología. Dirección electrónica: florenciastelzer@gmail.com

2 Investigadora Adjunta del Concejo Nacional de Investigaciones Científicas y Técnicas (CONICET) Argentina. Doctora en Psicología. Dirección electrónica: isabelintrozzi@gmail.com

${ }^{3}$ Becaria Post Doctoral del Concejo Nacional de Investigaciones Científicas y Técnicas (CONICET), Argentina. Doctora en Psicología. Dirección electrónica: marialauraandres@gmail.com

${ }^{4}$ Investigadora Adjunta del Concejo Nacional de Investigaciones Científicas y Técnicas (CONICET) Argentina. Doctora en Psicología. Dirección electrónica: mariamartarichards@gmail.com

${ }^{5}$ Investigador Independiente del Concejo Nacional de Investigaciones Cientificas y Técnicas (CONICET) Argentina. Doctor en Educación en el área de Psicología Educacional. Dirección electrónica: sebasurquijo@gmail.com

Artículo recibido: 4 de abril, 2017

Enviado a corrección: 10 de octubre, 2017

Aprobado: 13 de noviembre, 2017

Volumen 18 Número 1, Año 2018, ISSN 1409-4703 


\section{Introducción}

El nivel de conocimiento de matemática alcanzado predice las oportunidades laborales, el éxito profesional, el nivel de ingresos y el bienestar individual (Ancker y Kaufman, 2007; Every Child a Chance Trust, 2009; Grinyer, 2005; Paglin y Rufolo, 1990; Parsons y Bynner, 2005; Rivera-Batiz, 1992; Rose y Betts, 2004). Además, este conocimiento constituye uno de los pilares para el desarrollo científico-tecnológico y económico de los países, por lo que su aprendizaje tiene un importante impacto social (Hanushek y Woessmann, 2010; Parsons y Bynner, 2005). Desafortunadamente, distintas investigaciones indicaron que un porcentaje amplio de las niñas y los niños no alcanzan el nivel elemental de conocimiento matemático esperado para su nivel educativo, o adquieren únicamente las competencias matemáticas más básicas (Departamento de Evaluación de la Calidad Educativa [Diniece], 2013; Flotts et al., 2015; Organización para la Cooperación y el Desarrollo Económicos [OCDE], 2014). Por ejemplo, los resultados del PISA (OCDE, 2014) indicaron que el 32\% del estudiantado de todos los países y economías participantes no alcanzó el nivel de conocimiento básico establecido para el área de matemática en tal estudio. En un sentido similar, el $47 \%$ de los estudiantes de 6ำ año de los países participantes del Tercer Estudio Regional Comparativo y Explicativo (Terce) presentaron el nivel de desempeño más bajo y elemental convenido para el área de matemática en tal investigación (Flotts et al., 2015).

El conocimiento de la matemática muestra una estructura jerárquica donde el aprendizaje de las nociones matemáticas básicas posibilita el logro de conocimientos más avanzados (Geary et al., 2008; Jordan, Kaplan, Ramineni y Locuniak, 2009; Siegler et al., 2012). Un estudio realizado con una muestra representativa de estudiantes de Estados Unidos y Reino Unido (Siegler et al., 2012) reportó que, dentro de los contenidos curriculares del área de matemática de la educación primaria, las nociones de división y fracciones resultaban centrales para el aprendizaje de la matemática en la secundaria. Puntualmente, Siegler et al. (2012) indicaron que ambos conocimientos constituían los únicos predictores del aprendizaje del álgebra y del desempeño general en matemática en la secundaria cuando se consideraba también el efecto explicativo de otras variables académicas, sociales y cognitivas (e.g., capacidad de cálculo de adición, sustracción y multiplicación, memoria de trabajo, lectura, cociente intelectual, ingreso y ocupación familiar) (Siegler et al., 2012).

En la última década se han analizado los factores académicos y cognitivos que explican el conocimiento de las fracciones (Hecht y Vagi, 2010; Namkung y Fuchs, 2015; Siegler y Pyke, 2013; Vukovic et al., 2014), y las variables académicas (e.g., modo de 
enseñanza, conocimientos matemáticos previos) que intervienen en el aprendizaje de la división (Anghileri, Beishuizen, y Van Putten, 2002; Boero, Ferrari y Ferrero, 1989; Fagginger, 2016; Kratzer, y Willoughby, 1973; Van Putten, Van den Brom-Snijders y Beishuizen, 2005). No obstante, los factores cognitivos generales implicados en este último conocimiento han sido poco estudiados (Geary et al., 2008). Identificar dichos factores es muy relevante, dado que permitiría reconocer de manera temprana a niños y niñas con potenciales dificultades en el aprendizaje posterior de la matemática, y diseñar estrategias para prevenir tales obstáculos.

En el sistema educativo argentino, la enseñanza de la división comienza en $2^{\circ}$ año (niños de 7-8 años) y continúa durante toda la escolaridad primaria (Ministerio de Educación, Ciencia y Tecnología de Argentina, 2011a, 2011b). Inicialmente la división es introducida mediante problemas de proporcionalidad simple, rectangulares, de partición y reparto $\left(2^{\circ}\right.$ año), y es en $3^{\circ}$ año cuando comienza el trabajo sistemático hacia la construcción del algoritmo convencional de división para divisiones simples y complejas con divisores de un dígito (Ministerio de Educación, Ciencia y Tecnología, 2007a, 2007b). En la literatura existe cierto consenso en considerar como divisiones simples aquellas que involucran divisores de un dígito e implican dividendos que son múltiplos del divisor (e.g., 56/8) (LeFevre y Morris, 1999; Robinson et al., 2006). Por otra parte, las divisiones complejas involucran dividendos de un dígito que no constituyen múltiplos del divisor (e.g., 49/3) y operaciones que involucran divisores y/o cocientes de dos o más dígitos (e.g., 360/20) (Hickendorff, van Putten, Verhelst y Heiser, 2010). No obstante, es importante aclarar que el carácter complejo de la operación reside en la dificultad cognitiva que su resolución demanda conforme al conocimiento aritmético esperado para el nivel educativo del participante. Por ejemplo, 100/20 constituiría una división compleja para un estudiante de $3^{\circ}$ año, mientras que se consideraría como un cálculo simple para un estudiante de 6ำ año.

En $4^{\circ}$ año se amplia y profundiza la enseñanza de esta operación. Puntualmente, se introducen las divisiones con divisores de dos cifras, y se incentiva al estudiantado abstraer relaciones numéricas para el cálculo mental de operaciones de división (e.g., Si 60/10=6, ¿cuánto será 60/30 y 600/20?, ¿Por qué?) (Ministerio de Educación, Ciencia y Tecnología de Argentina, 2007c). Además, se acentúa el trabajo que apunta a la comprensión del algoritmo convencional de división, lo cual requiere recuperar el conocimiento de las otras operaciones de cálculo aritmético y utilizarlo de forma secuencial y controlada en los diferentes pasos de solución de problemas de división (Ministerio de Educación, Ciencia y Tecnología de 
Argentina, 2007c). Dado que $4^{\circ}$ año representa un salto importante en el nivel de complejidad del conocimiento de la división exigido, la comprensión de los factores que explican este conocimiento en dicho año escolar reviste un particular interés.

En función de lo mencionado respecto de los contenidos de división previstos en el diseño curricular de $4^{\circ}$ año, sería esperable que el conocimiento de esta operación se relacione con dos grandes factores cognitivos; a saber: la capacidad de control cognitivo (memoria de trabajo e inhibición) y la inteligencia fluida. El objetivo de este trabajo es analizar si la capacidad de estudiantes de $4^{\circ}$ año de realizar operaciones de división se relaciona con tales factores cognitivos.

\subsection{Antecedentes previos sobre los factores cognitivos propuestos}

\subsubsection{Inhibición}

La inhibición constituye un proceso cognitivo que permite el control en el procesamiento consciente de la información a través de la supresión de la información irrelevante para el logro de metas. Este proceso impide la captura del foco de atención por distractores, lo cual posibilita el procesamiento consciente y controlado de la información clave para el logro de objetivos (Diamond, 2013). Ciertos estudios sugirieron que fallas en la inhibición explicarían las dificultades en aritmética de estudiantes con un desarrollo matemático atípico (D'Amico y Passolunghi, 2009; van der Sluis, de Jong y van der Leij, 2004). Además, este proceso ha sido vinculado a la capacidad general de cálculo aritmético en estudiantes con un desarrollo matemático típico (Gilmore et al., 2013; Navarro et al., 2011). Sin embargo, otras investigaciones no corroboraron la relación de la inhibición con el cálculo aritmético en niños con un desarrollo matemático atípico (Censabella y Noël, 2005; 2007) o típico (Bellon, Fias y De Smedt, 2016). Los desacuerdos entre estudios respecto del rol de la inhibición en el cálculo aritmético podrían deberse a que estos consideraron participantes de diferentes años escolares y evaluaron el cálculo aritmético utilizando un índice general del desempeño en diversas operaciones (adición, sustracción, etc.).

En la literatura se ha sugerido que la relación de procesos de control cognitivo con el conocimiento de la matemática variaría conforme al tipo de conocimiento y el nivel de aprendizaje del mismo (Cragg y Gilmore, 2014; Raghubar, Barnes y Hecht, 2010). Por lo tanto, sería posible que la inhibición se relacione con la capacidad de división de forma diferente respecto de otras operaciones aritméticas en estudiantes de distintos años escolares. En apoyo parcial a esta hipótesis, Siegler y Pyke (2013) hallaron que la inhibición 
se vinculaba con la capacidad de división en estudiantes de $6^{\circ}$ año, pero no así en estudiantes de $8^{\circ}$ año.

En el contexto áulico suelen presentarse diferentes distractores, por lo que la capacidad de inhibir la captura atencional por los mismos y focalizar la atención sobre la información relevante sería clave para el éxito académico. Por lo anterior consideramos que la inhibición se relacionará con la capacidad de cálculo de operaciones de división en estudiantes de $4^{\circ}$ año. Tal como ha sido mencionado, este año escolar representa un salto importante en el nivel de complejidad del conocimiento de la división exigido, por lo que anticipamos que la capacidad de suprimir la interferencia de distractores facilitará la focalización de la atención en clase y un mejor aprendizaje de este contenido.

\subsubsection{Memoria de trabajo}

La memoria de trabajo (MT) constituye un sistema de capacidad limitada que interviene en la retención y el procesamiento concurrente y controlado de la información (Baddeley, 2012). Uno de los modelos de MT más aceptados distingue sistemas de memoria independientes para la retención temporaria de la información verbal y visoespacial (Baddeley y Hitch, 1974; Baddeley, 2012). Sobre estos sistemas intervendría un sistema de control atencional amodal, designado ejecutivo central, responsable de la focalización y división atencional, actualización, monitoreo y manipulación de la información retenida en los sistemas verbal y visoespacial (Baddeley, 2012). Es decir, la MT implica la retención temporaria de información verbal o visoespacial y su concurrente procesamiento controlado (e.g., actualización, monitoreo, etc.). Si bien el ejecutivo central es amodal, algunos estudios sugieren que las tareas de MT que involucran material verbal y visoespacial explican de manera diferente el conocimiento de la matemática (Jarvis y Gathercole, 2003; Raghubar et al., 2010; Van de Weijer-Bergsma, Kroesbergen, y Van Luit, 2015), por lo que distinguiremos, en consonancia con la literatura, una MT verbal y visoespacial, según el formato de la información retenida. En estudios previos se reportó una relación de ambas formas de MT con la capacidad de división en estudiantes entre 3ํy y 6año (Hansen et al., 2015; Siegler y Pyke, 2013; Van Der Ven, Van Der Maas, Straatemeier y Jansen, 2013; Van de WeijerBergsma et al., 2015). Se ha sugerido que la MT visoespacial contribuiría al cálculo aritmético facilitando el uso de estrategias visoespaciales - tales como la representación de las magnitudes en la recta numérica - para la estimación de los resultados (Van Der Ven et al., 2013). Por otro lado, se ha propuesto que la MT verbal intervendría en el cálculo de la división al permitir el control, actualización y monitoreo de la información numérica en la 
ejecución de las estrategias de cálculo mental y algorítmico.

Si bien los resultados respecto del rol de la MT en el cálculo de la división son consistentes, estos provienen de estudios efectuados con participantes insertos en sistemas educativos anglosajones y europeos (Hansen et al., 2015; Siegler y Pyke, 2013; Van Der Ven et al., 2013; Van de Weijer-Bergsma et al., 2015). Investigaciones previas indicaron que el diseño curricular y los materiales didácticos utilizados durante la instrucción formal en aritmética afectan el uso de estrategias para el cálculo divisorio de los estudiantes (Anghileri, 2001; Kratzer y Willoughby, 1973). Considerando que la carga de retención y procesamiento de la MT varía en diferentes estrategias (Geary et al., 2008), sería posible que el efecto de la capacidad de MT sobre el cálculo de la división varíe en estudiantes de diferentes sistemas educativos. Por lo anterior, identificar si la MT se vincula a la capacidad de división en estudiantes de Argentina representa un aporte al conocimiento preexistente.

\subsubsection{Inteligencia fluida}

La inteligencia fluida refiere a la capacidad de razonamiento abstracto y solución de problemas de manera independiente al conocimiento previo (Cattell, 1987). Involucra la capacidad de identificar y procesar patrones de relaciones abstractas entre representaciones espaciales, verbales o numéricas, con el objeto de solucionar problemas. Tal inteligencia tiene un rol central en la resolución de problemas novedosos, por lo cual se constituye como esencial a la hora de la formación y consolidación de nuevos conocimientos y habilidades (Ferrer, O' Hare y Bunge, 2009).

Diferentes investigaciones indicaron que la inteligencia fluida predice el desempeño general en matemática (Aragón, Delgado, Aguilar, Araujo y Navarro, 2013; Ferrer y McArdle, 2004; McArdle, Hamagami, Meredith y Bradway, 2000; McArdle, Ferrer-Caja, Hamagami y Woodcock, 2002). Pese a ello, se encuentran contradicciones respecto de la contribución de esta habilidad en la capacidad general de cálculo aritmético (e.g., Fuchs et al., 2006; Peng et al., 2016; Stock, Desoete y Roevers, 2009) ya que su relación específica con el cálculo de la división no ha sido analizada.

La elaboración de procedimientos de cálculo de división exige la representación y el procesamiento de la relación numérica entre divisor y dividendo. Además, la verificación del resultado obtenido requiere considerar las relaciones entre divisor, dividendo, cociente y resto. Como fue mencionado, en el sistema educativo argentino se prevé que el cálculo de operaciones de división evolucione en $4^{\circ}$ año desde estrategias que involucran múltiples pasos (e.g., restas sucesivas del divisor al dividendo) hasta la construcción de 
procedimientos más económicos (Ministerio de Educación, Ciencia y Tecnología de Argentina, 2011b). Esto requiere que, en función del objetivo de cálculo perseguido (exacto o aproximado), los estudiantes consideren las relaciones numéricas involucradas en un problema y elaboren un procedimiento apropiado de solución. La construcción de estrategias más económicas resulta clave para la solución de divisiones en $4^{\circ}$ año, ya que en ciertos problemas la ejecución de múltiples pasos podría conducir a resultados incorrectos producto de su extensión (e.g., utilizar como estrategia la adición sucesiva del divisor para resolver 1200 /30). El desarrollo de estrategias más económicas exige que el estudiantado sea capaz de identificar, abstraer y procesar las relaciones numéricas implicadas en diferentes problemas, por lo que anticipamos que la inteligencia fluida se relacionará con la capacidad de cálculo de división en estudiantes de $4^{\circ}$ año de la escolaridad primaria.

\subsection{Contribución de este estudio a la literatura}

Diferentes autores sugirieron que la relación de procesos de control cognitivo con el conocimiento de la matemática varía conforme al tipo de conocimiento y al nivel de aprendizaje del mismo (Cragg y Gilmore, 2014; Raghubar et al., 2010). El aprendizaje de la división constituye un proceso complejo que insume varios años de la escolaridad básica. En 4ํaño se incrementa notoriamente la complejidad del contenido de división trabajado, por lo que determinar el efecto de procesos y habilidades cognitivas sobre el desempeño en división en este año, es clave para identificar a estudiantes con posibles dificultades en el dominio de este conocimiento y diseñar estrategias para prevenirlas

Si bien existe evidencia de un vínculo de la inhibición, la MT y la inteligencia fluida con el conocimiento de matemática, la relación específica de estos procesos con la capacidad de división en estudiantes $4^{\circ}$ año del sistema educativo argentino no ha sido abordada. De este modo, este estudio contribuye a la literatura al analizar si la capacidad de cálculo de división en estudiantes de tal año escolar se asocia con tales factores cognitivos.

\section{Metodología}

\subsection{Diseño}

Se efectuó una investigación cuantitativa, con un diseño no experimental transversal correlacional. Este diseño permite analizar relaciones transversales entre los factores cognitivos considerados y la capacidad de realizar operaciones de división. 


\subsection{Participantes}

La muestra fue seleccionada de manera no probabilística, por disponibilidad, y estuvo integrada por 175 niños asistentes a 4ํaño del segundo ciclo de la educación básica de tres establecimientos educativos de gestión pública y uno de gestión privada ubicados en zonas urbanas de la ciudad de Mar del Plata, Argentina. Los mismos recibían instrucción en matemática conforme a los lineamientos didácticos sugeridos desde el Ministerio de Educación de Argentina (Ministerio de Educación, Ciencia y Tecnología de Argentina, 2007c). Fueron excluidos del análisis de los datos aquellos casos que: (a) presentaban diagnóstico previo de trastornos neurológicos o psiquiátricos, (b) tenían un peso de nacimiento inferior a 2,5 kilos o gestación inferior a las 37 semanas, (c) se encontraban en etapa de diagnóstico o tratamiento psicológico/psicopedagógico, o (d) mostraron una puntuación en la escala coloreada del Test Matrices Progresivas de Raven (1989) correspondiente a la categoría deficiente según los baremos definidos por Cayssials (1993).

Trabajos previos mostraron una relación entre la presencia de alguna de estas condiciones y el desempeño en matemática (Hudson, Browder y Wood, 2013; Mayes Dickerson y Calhoun, 2006; McCormick, Litt, Smith y Zupancic, 2011), por lo que la exclusión de participantes con dichas características permite controlar el efecto de variables extrañas en la estimación de las relaciones entre las variables de interés en esta investigación. La muestra final quedó integrada por 135 niños (69 varones) de diferente estatus social (18.1\% bajo; $32.8 \%$ medio-bajo; $15.5 \%$ medio; $20.7 \%$ medio-alto; $12.9 \%$ alto).

\subsection{Instrumentos}

\subsubsection{Instrumentos de medición de las variables bajo estudio}

\subsubsection{Inteligencia fluida}

Para su medición se utilizó la Escala Coloreada del Test de Matrices Progresivas (Raven, 1989). Dicha escala está compuesta por 36 láminas que muestran matrices gráficas con una pieza faltante. Se solicita a los participantes que identifiquen entre seis opciones aquella que completa la matriz. Esta tarea exige la resolución de problemas a través de la comparación de formas y razonamiento por analogía. La escala coloreada está integrada por tres series $(A, A b, B)$ de 12 láminas cada una. La serie $A$ se compone de problemas simples, de tipo perceptuales para cuya resolución el razonamiento por analogía no resulta imprescindible. La serie Ab evalúa la capacidad de percibir figuras discretas como todos relacionados espacialmente. La serie B se caracteriza por exigir el razonamiento por 
analogía. Los participantes reciben un punto por cada problema resuelto correctamente. La confiabilidad estimada a través del Alpha de Cronbach fue $\alpha .79$

\subsubsection{Inhibición}

Se utilizó la tarea de Búsqueda Visual Conjunta (TBVC). Esta constituye una adaptación del Paradigma de Búsqueda Visual Conjunta de Treisman y Gelade (1980). En dicha tarea el participante debe identificar la presencia o ausencia de un estímulo target (cuadrado azul) que se presenta mezclado entre un conjunto de distractores similares que comparten una de las dos características visuales que definen el estímulo target: color (círculos azules) o forma (cuadrados rojos). El tamaño de los cuadrados es de $8 \mathrm{~cm}$ por lado y los círculos de $8 \mathrm{~cm}$ de diámetro. Los distractores, al compartir un atributo con el target (forma o color), garantizan la semejanza visual entre los estímulos y el efecto de interferencia necesario para la activación de la inhibición. La tarea está compuesta por un bloque de 10 ensayos de práctica, seguido de tres bloques de evaluación de 40 ensayos cada uno. En cada ensayo el participante debe presionar dos teclas diferentes ( $Z$ y $M$ ) en función de la presencia o ausencia del estímulo target. En cada bloque, los ensayos se distribuyen en cuatro condiciones definidas por la cantidad de distractores (4, 8, 16 y 32). En cada ensayo la mitad de los distractores son círculos azules y la otra mitad cuadrados rojos. Las condiciones se distribuyen de manera aleatoria por bloque. Se consideró como indicador del desempeño el TR medio calculado sobre las respuestas correctas en la condición de 32 distractores (condición de mayor interferencia y mayor exigencia atencional). Estudios previos indicaron que esta tarea presenta adecuados índices de confiabilidad y validez, tanto en adultos (Introzzi, Andrés, Canet-Juric, Stelzer, y Richard's, 2016) como en niños (ver Richard's, Introzzi, Zamora y Vernucci, 2017; Richard's et al., 2017).

\subsubsection{Memoria de trabajo verbal}

Se empleó la tarea de memoria de trabajo dual verbal. Constituye una tarea informatizada que evalúa la capacidad de retención y procesamiento concurrente de la información a través de la ejecución de dos tareas, una primaria y otra secundaria. La tarea primaria exige recordar series de dígitos que se presentan durante un segundo en la pantalla del ordenador, de uno en uno, escritos en diferentes colores. La tarea secundaria requiere que los participantes mencionen en voz alta el color en el que apareció cada dígito tras su desaparición. El número de dígitos a retener se incrementa de a una unidad tras el recuerdo correcto de la secuencia de dígitos mostrada. Asimismo, la prueba se interrumpe luego de 
dos errores consecutivos. Se consideró como indicador de la MT verbal la cantidad máxima de dígitos recordados. Estudios previos indicaron que esta tarea presenta niveles de validez y confiabilidad adecuados para su aplicación en niños (Canet-Juric, Introzzi y Burin, 2015).

\subsubsection{MT visoespacial}

Se administró la tarea de memoria de trabajo dual visoespacial. Al igual que la tarea de MT verbal, constituye una tarea informatizada que demanda la realización de una tarea primaria y otra secundaria. La tarea primaria exige recordar la secuencia de aparición de cruces de diferentes colores presentadas durante un segundo, de una en una, sobre una matriz 4x4. La tarea secundaria requiere que los participantes indiquen el color de cada cruz, tocando o señalando con el cursor una paleta de colores colocada a la derecha de la matriz, tras la desaparición de cada cruz. El número de localizaciones a recordar se incrementa a una unidad tras el recuerdo correcto de la secuencia de localizaciones mostrada. Además, la tarea se interrumpe tras dos ensayos sucesivos incorrectos. Se consideró como indicador de la MT visoespacial la cantidad máxima de localizaciones recordadas. Estudios previos indicaron que esta tarea presenta niveles de validez y confiabilidad adecuados para su aplicación en niños y niñas (Canet-Juric et al., 2015).

\subsubsection{Capacidad de división}

Constituye una adaptación de la tarea implementada por Hansen et al. (2015) y Siegler

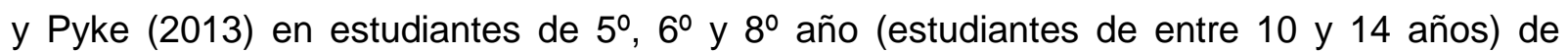
Estados Unidos. Esta fue adaptada conforme al nivel de aprendizaje de las operaciones de división esperado para los estudiantes de $4^{\circ}$ año según los núcleos de aprendizaje prioritarios del sistema educativo argentino (Ministerio de Educación, Ciencia y Tecnología de Argentina, 2011b). La misma consiste en una prueba de lápiz y papel donde se presentan de manera consecutiva y en líneas independientes seis divisiones de complejidad creciente (9/3; 10/4; 56/8; 180/60; 1200/400; 306/9). Los estudiantes deben resolver la mayor cantidad de divisiones en el menor tiempo posible, pudiendo utilizar para ello cualquier estrategia de cálculo (e.g, recuperación mnémica del resultado, descomposición, aplicación del algoritmo convencional, etc.). Para ello disponen de un tiempo máximo de 15 minutos. Los estudiantes reciben dos puntos por cada respuesta correcta y un punto cuando la respuesta es correcta pero se detecta un error en el procedimiento de cálculo. La puntuación máxima posible es 12. La confiabilidad se estimó a través del coeficiente Alpha de Cronbach ( $\alpha$.72). 
2.3.2. Instrumentos para el control de variables extrañas y caracterización de la muestra

\subsubsection{Ficha Sanitaria}

Consiste en un cuestionario de informe paterno donde se indagaron las variables (a) meses de gestación alcanzados, (b) peso de nacimiento (c) diagnóstico de trastornos neurológicos o psiquiátricos previos al desarrollo del estudio (d) evaluación diagnóstica o tratamiento psicológico o psicopedagógico en curso.

\subsubsection{Cuestionario de Estatus Social (ES)}

Constituye un cuestionario semi-estructurado de informe paterno que permite calcular el ES a través de los indicadores nivel educativo y tipo de ocupación del principal sostén económico del niño o niña. El nivel educativo se codificó en una escala de siete puntos que toma como referencia las características del sistema educativo argentino (1=educación primaria incompleta; 2=educación primaria completa; 3=educación secundaria incompleta; 4=educación secundaria completa; 5=educación universitaria incompleta; $6=$ =educación universitaria completa y $7=$ =educación de posgrado completa 0 incompleta) (Pascual, Galperín y Bornstein, 1993). El tipo de ocupación se clasificó conforme a una adaptación realizada por el grupo de investigación de la Escala de Grupos Ocupacionales elaborada para la sociedad Argentina por Sautú (1989). La escala utilizada considera cinco categorías a las que se les asignan diferentes puntuaciones (1.5=trabajadores sub-calificados; $3.5=$ trabajadores calificados y semi-calificados; $5.5=$ empleados administrativos $y$ cuentapropistas; $6.5=$ técnicos y semi-profesionales y $8.5=$ profesionales y administrativos de mayor rango).

Se computó el ES según el índice de Hollingshead (2011) multiplicando los valores del nivel educativo (NE) y ocupacional (NO) por unos factores de corrección (FC), y sumando los valores obtenidos en un puntaje total $(E S=[N E \times F C]+[N O \times F C])$. Este puntaje se puede clasificar en categorías indicadoras del ES: alto: (55- 63,5), medio-alto (40-54), medio (3039), medio-bajo (20-29) y bajo (0-19). Este índice se ha utilizado en distintos contextos socioculturales para la medición del ES (Adams y Weakliem, 2011) incluyendo el latinoamericano y nacional (e.g., Andrés, Castañeiras y Richaud de Minzi, 2014; Andrés, Richaud de Minzi, Castañeiras, Canet-Juric y Rodríguez-Carvajal, 2016; Piccinini, Tudge, Marín, Bitencourt-Frizzo y Cássia-Sobreira Lopes, 2010). 


\subsection{Procedimiento}

Se informó a las autoridades de las instituciones educativas sobre los objetivos y procedimientos de la investigación. Una vez que dieron su aprobación se solicitó a los padres la autorización para que sus hijos participaran a través de la firma de un consentimiento informado. Se informó a los niños del estudio y se evaluó a aquellos que contaban con el consentimiento informado de sus padres y manifestaron su intención de participar. Los niños fueron evaluados durante el horario escolar, dentro de los establecimientos educativos a los que asistían, en aulas asignadas por éstos exclusivamente para tal propósito. Las tareas de MT verbal, MT visoespacial e inhibición se administraron en una única sesión de evaluación individual por evaluadores entrenados. Estos evaluadores eran estudiantes avanzados de la carrera de Psicología y fueron capacitados por parte de uno de los responsables del equipo de investigación en la administración de las diferentes tareas. El tiempo total aproximado de administración por participante fue de 40 minutos.

El orden de administración de las tareas fue aleatorizado entre los distintos participantes. Las evaluaciones en inteligencia fluida y división se administraron en dos sesiones de evaluación independientes, de forma grupal en el salón habitual de clase de los estudiantes por uno de los investigadores responsables del proyecto. Las preguntas del estatus social y la ficha sanitaria fueron enviadas a los padres a través del cuaderno de comunicaciones. Previo a la evaluación de la capacidad de división se verificó en una entrevista con las docentes que los estudiantes habían realizado durante el trabajo escolar operaciones de división de igual complejidad a aquellas que componían la tarea y habían recibido instrucción docente en alguna estrategia posible de resolución de tales operaciones.

\section{Resultados}

\subsection{Análisis descriptivos}

Los datos fueron analizados utilizando el software estadístico SPSS versión 19. En primer lugar se realizaron análisis descriptivos para conocer la distribución de los valores de las variables bajo estudio (ver Tabla 1). La Tabla 1 muestra los valores mínimos, máximos, media, desviación estándar y el estadístico Kolmogorov-Simirnov correspondiente a las variables bajo estudio. 
Tabla 1

Estadísticos descriptivos de las variables de estudio

\begin{tabular}{|l|r|r|r|r|r|}
\hline \multicolumn{1}{|c|}{ Variable } & \multicolumn{1}{c|}{ Mínimo } & \multicolumn{1}{c|}{ Máximo } & \multicolumn{1}{c|}{ M } & \multicolumn{1}{c|}{ DE } & \multicolumn{1}{c|}{ Kolmogorov-Simirnov } \\
\hline División & 0 & 12 & 4.75 & 3.39 & $1.62^{*}$ \\
\hline Inteligencia fluida & 24 & 36 & 29.78 & 3.24 & 1.16 \\
\hline MT verbal & 0 & 5 & 3.17 & .82 & $3.18^{* *}$ \\
\hline MT visoespacial & 0 & 7 & 3.48 & 1.43 & $1.89^{*}$ \\
\hline Inhibición (ms) & 661 & 3572 & 1773.11 & 441.96 & $1.38^{*}$ \\
\hline
\end{tabular}

Notas ${ }^{*} \mathrm{p}<.05,{ }^{*} \mathrm{p}<.01$

Fuente: Elaboración propia del autor. Datos correspondientes a estudiante de $4^{\circ}$ año del nivel primario de Argentina evaluados durante el primer cuatrimestre del ciclo lectivo 2016.

Conforme a la distribución de las puntuaciones observadas en la tarea de división, se clasificó a los estudiantes en tres grupos de desempeño en división, aquellos que presentaban un desempeño bajo (puntuaciones inferiores o iguales al percentil 25), aquellos que mostraban un desempeño medio (puntuaciones entre el percentil 26 y el percentil 74) y los que mostraron un desempeño alto (puntuaciones iguales o superiores al percentil 75). Esta agrupación fue considerada ya que permitía distinguir entre: (a) aquellos estudiantes que presentaron grandes dificultades en la realización de operaciones de división y no pudieron resolver ninguno o solo uno de los seis problemas de división administrados (grupo de desempeño bajo), (b) aquellos participantes que fueron capaces de realizar algunos de los cálculos administrados (dos o tres de los seis cálculos, grupo de desempeño medio) y (c) aquellos que pudieron realizar la mayor parte de los ejercicios (cuatro o más cálculos, grupo de desempeño alto). En la Tabla 2 se presentan los valores mínimos, máximos, media, desviación estándar y el estadístico Kolmogorov-Smirnov para cada grupo en los diferentes factores cognitivos estudiados. 
Tabla 2

Estadísticos descriptivos de los factores cognitivos estudiados en los distintos grupos de desempeño

\begin{tabular}{|c|c|c|c|c|}
\hline Factor cognitivo & Estadístico & $\begin{array}{c}\text { Grupo D. Bajo } \\
(n=46)\end{array}$ & $\begin{array}{c}\text { Grupo D. Medio } \\
(n=51)\end{array}$ & $\begin{array}{c}\text { Grupo D. Alto } \\
(n=35)\end{array}$ \\
\hline \multirow{5}{*}{ Inteligencia fluida } & Mínimo & 24 & 25 & 24 \\
\hline & Máximo & 35 & 35 & 36 \\
\hline & $\mathrm{M}$ & 28.67 & 30.31 & 30.65 \\
\hline & $\mathrm{DE}$ & 3.31 & 2.85 & 3.36 \\
\hline & $\begin{array}{l}\text { Kolmogorov- } \\
\text { Simirnov }\end{array}$ & 1.19 & .89 & .65 \\
\hline \multirow{5}{*}{ MT verbal } & Mínimo & 2 & 2 & 2 \\
\hline & Máximo & 4 & 5 & 5 \\
\hline & $M$ & 2.87 & 3.33 & 3.37 \\
\hline & $\mathrm{DE}$ & .62 & .71 & .91 \\
\hline & $\begin{array}{l}\text { Kolmogorov- } \\
\text { Simirnov }\end{array}$ & $2.13^{\star \star}$ & $2.06^{\star *}$ & $1.36^{*}$ \\
\hline \multirow{5}{*}{ MT visoespacial } & Mínimo & 0 & 0 & 0 \\
\hline & Máximo & 5 & 6 & 7 \\
\hline & M & 3.09 & 3.48 & 3.94 \\
\hline & $\mathrm{DE}$ & 1.41 & 1.4 & 1.39 \\
\hline & $\begin{array}{l}\text { Kolmogorov- } \\
\text { Simirnov }\end{array}$ & 1.14 & 1.02 & 1.03 \\
\hline \multirow{5}{*}{ Inhibición (TR ms) } & Mínimo & 1169 & 1295 & 661 \\
\hline & Máximo & 2608 & 2786 & 3033 \\
\hline & $\mathrm{M}$ & 1747.55 & 1797.58 & 1637.37 \\
\hline & $\mathrm{DE}$ & 316.37 & 368.69 & 454 \\
\hline & $\begin{array}{l}\text { Kolmogorov- } \\
\text { Simirnov }\end{array}$ & .95 & 1.12 & 1.20 \\
\hline
\end{tabular}

Notas. ${ }^{*} p<.01 ;{ }^{* *} p<.001$

Fuente: Elaboración propia del autor. Datos recolectados en estudiante de $4^{\circ}$ año del nivel primario de Argentina evaluados durante el primer cuatrimestre del ciclo lectivo 2016.

A fin de establecer si los estudiantes con distinto nivel de desempeño en división diferían entre sí en los distintos factores cognitivos estudiados, se efectuaron comparaciones entre los grupos de desempeño. Para esto, en primer lugar se efectuó un análisis de correlación de Pearson para evaluar el cumplimiento del supuesto del MANOVA de correlaciones moderadas entre las variables dependientes. Tal como se observa en la Tabla 3, los factores cognitivos mostraron correlaciones bajas entre sí, por lo que se efectuaron ANOVAS de un factor para cada uno de ellos. Se verificó, a través de las pruebas de Levene, Kolgomorov-Smirnov y el análisis de las distribuciones gráficas, el cumplimiento de los supuestos del ANOVA de homogeneidad de varianzas y normalidad de las distribuciones entre grupos. Tales supuestos se cumplieron para todas las variables de estudio exceptuando la MT verbal. En esta última no se cumplió ninguno de tales supuestos, por lo que se aplicó un análisis no paramétrico utilizando la prueba $U$ de Mann Whitney. 
Tabla 3

Correlaciones entre los factores cognitivos

\begin{tabular}{|l|c|c|c|}
\hline \multicolumn{1}{|c|}{ Factor cognitivo } & MT verbal & MT visoespacial & Inhibición \\
\hline Inteligencia fluida & $.26^{* *}$ & $.26^{* *}$ & -.02 \\
\hline MT verbal & & $.19^{*}$ & -.13 \\
\hline MT visoespacial & & & $-.23^{* *}$ \\
\hline
\end{tabular}

Notas, ${ }^{*} \mathrm{p}<.05 ;{ }^{* *} \mathrm{p}<.01$

Fuente: Elaboración propia del autor. Datos recolectados en estudiantes de $4^{\circ}$ año del nivel primario de Argentina evaluados durante el primer cuatrimestre del ciclo lectivo 2016.

\subsection{Comparación de los grupos de desempeño en división respecto de los factores} cognitivos estudiados

Se hallaron diferencias estadísticamente significativas entre los grupos de desempeño en división en inteligencia fluida $\left[F(2,125)=4.64, p<.05, \eta^{2}=.07\right]$, MT verbal $[H(2)=11.40$, $p<.01]$ y MT visoespacial $\left[F(2,126)=3.59, p<.05, \eta^{2}=.05\right]$. Por el contrario, no se observaron diferencias estadísticamente significativas entre los grupos en inhibición $[F(2,126)=1.89$, $\left.p=.16, \eta^{2}=.03\right]$.

Las comparaciones post hoc de Bonferroni indicaron que el grupo de estudiantes con un desempeño bajo en división, presentaba puntuaciones medias significativamente más bajas $(p<.05)$ en inteligencia fluida $(M=28.67, D E=3.31)$ respecto de los grupos de estudiantes con desempeño medio $(M=30.31, D E=2.85)$ y alto $(M=30.65, D E=3.36)$. Sin embargo, los grupos de desempeño medio y alto en división no diferían significativamente entre sí en inteligencia fluida.

Por otra parte, respecto de las diferencias significativas halladas en MT verbal, se contrastaron las diferencias entre grupos utilizando la prueba $U$ de Mann Whitney. Los resultados indicaron que el grupo de estudiantes con un desempeño bajo presentaba puntuaciones significativamente más bajas en $\mathrm{MT}$ verbal $(M d n=3)$ respecto de los grupos de desempeño medio $(M d n=3)(U=756, p<.01, r=-.32)$ y alto en división $(U=540, p<.01, r=-.29)$. No obstante, los grupos de desempeño medio $(M d n=3)$ y alto $(M d n=3)$ no diferían significativamente entre sí en MT verbal $(U=878, p=.89)$.

Por último, respecto de las diferencias entre grupos en MT visoespacial, las comparaciones post hoc de Bonferroni mostraron que los estudiantes con un desempeño bajo en división presentaban puntuaciones medias significativamente $(p<.05)$ inferiores en MT visoespacial $(M=3.09, D E=1.41)$, respecto del grupo de estudiantes con un desempeño alto en división $(M=3.94, D E=1.39)$. Por el contrario, los estudiantes con un desempeño bajo no diferían significativamente en su capacidad de MT visoespacial respecto de los 
estudiantes con un desempeño medio en división $(M=3.48, D E=1.4)(p=.55)$. Asimismo, los grupos de desempeño medio y alto en división no diferían significativamente entre sí en la capacidad de MT visoespacial $(p=.41)$.

\section{Conclusiones}

El objetivo de este trabajo fue analizar la relación de la capacidad de cálculo de división con la inteligencia fluida, la MT y la inhibición en estudiantes de $4^{\circ}$ año de la escolaridad primaria. Nuestros resultados indicaron que los estudiantes con distinto nivel de desempeño en división diferían entre sí en su capacidad de MT e inteligencia fluida. A pesar de esto, no observamos diferencias estadísticamente significativas entre ellos en su capacidad de inhibición.

Respecto de las diferencias entre grupos en la capacidad de MT, hallamos que los estudiantes con un desempeño bajo en división presentaban una menor capacidad de MT verbal respecto de aquellos con un desempeño medio y alto. Asimismo, los estudiantes con un desempeño bajo en división mostraban puntuaciones significativamente inferiores en MT visoespacial respecto de los estudiantes con un desempeño alto en este conocimiento. Sin embargo, los estudiantes con un desempeño medio y alto en división no diferían significativamente entre sí en su capacidad de MT verbal y visoespacial.

La relación hallada entre la MT verbal y el cálculo de la división se corresponde con lo reportado en estudios previos en estudiantes de otros sistemas educativos (Hansen et al., 2010; Siegler y Pyke, 2013; Van Der Ven et al., 2013; Van de Weijer-Bergsma et al., 2015). A excepción de los problemas de división cuyos cocientes han sido memorizados, el cálculo de esta operación requiere realizar secuencialmente e integrar los productos de diferentes operaciones. Esto exige el procesamiento controlado, monitoreo y actualización de la información numérica en la mente. En nuestro estudio observamos que los estudiantes utilizaban diferentes procedimientos informales para la resolución de las operaciones de división. Estos procedimientos coinciden con algunas de las estrategias de cálculo informal señaladas en la literatura (e.g., sustracción sucesiva del divisor al dividendo [180/60=180$60=120-60=60-60=0 "]$, utilización del conocimiento de múltiplos [9/3;3×3=9], partición del dividendo $[1200 / 400=800 / 400+400 / 400]$ o combinaciones de las anteriores) (Anghileri, et al., 2002; Hickendorff et al., 2010; Fagginger, 2016). Todos estos procedimientos requieren el control y el monitoreo en el procesamiento de la información numérica. Además, algunos estudiantes utilizaban el algoritmo tradicional de división. Este demanda integrar 
secuencialmente los productos de las diferentes operaciones aritméticas desarrolladas. De este modo, la exigencia de retención y procesamiento concurrente de información numérica en el cálculo de operaciones de división explicaría que los estudiantes con un desempeño bajo en división muestren una menor capacidad de MT verbal respecto de aquellos con un desempeño medio y alto.

Desde otra perspectiva, nuestros resultados también coinciden con lo señalado en investigaciones previas respecto a la relación de la MT visoespacial con la capacidad de cálculo de operaciones de división (Van Der Ven et al., 2013; Van de Weijer-Bergsma et al., 2015). En la literatura se sugirió que el uso de representaciones visoespaciales facilitaría el procesamiento de las relaciones numéricas involucradas en problemas de cálculo y, consecuentemente, su solución (Geary et al., 2008; Van Der Ven et al., 2013; Meyer, Salimpoor, Wu, Geary y Menon, 2010). Por ejemplo, se ha propuesto que la representación en la recta numérica de las magnitudes de un problema, permitiría estimar y calcular el resultado de diferentes operaciones aritméticas (Geary et al., 2008). Asimismo, se ha sugerido que la organización de la información numérica en columnas durante la ejecución de algoritmos de cálculo y la comprensión de los conceptos de "préstamo" y "carga" durante su ejecución, involucran el procesamiento de relaciones visoespaciales y dependen de la MT visoespacial (Bull, Espy y Wiebe, 2008). De este modo, el uso de habilidades y estrategias visoespaciales para el procesamiento de las magnitudes implicadas en operaciones de división, podría explicar las diferencias observadas entre los estudiantes con un desempeño bajo y alto en división.

Otra posible explicación de nuestros resultados es que la relación observada de la MT verbal y visoespacial con el cálculo de división, se origine en el componente de control, actualización y monitoreo de la información implicado en ambas formas de MT (ejecutivo central). Es decir, que la relación sea independiente del modo de representación de la información (verbal o visoespacial). Sin embargo, la correlación de las puntuaciones en ambas tareas de MT es baja $(r=.19)$, lo que sugiere que tales tareas evalúan componentes independientes de este constructo, que se asocian de forma diferente con la capacidad de división.

Por otra parte, respecto de las diferencias en inteligencia fluida entre los grupos con diferente capacidad de cálculo de división, estas podrían deberse a la exigencia de reconocimiento e integración de relaciones numéricas implicada en esta operación. Además, otra posible explicación complementaria a la anterior, es que tales diferencias se deban al 
modo de enseñanza de la división sugerida desde organismos oficiales de Argentina, e implementada en los establecimientos educativos participantes. En los materiales didácticos avalados desde organismos oficiales de Argentina se proponen actividades que fomentan el reconocimiento, la abstracción y la aplicación de relaciones numéricas para la solución mental de cálculos de división (e.g., "Sabiendo que 1600/10=160, sin hacer las cuentas averigua cuánto es 1600/20 y 1600/40") (Broitman, Ecobar, Etchemendy, Novembre y Sancha, 2011, p. 50). Igualmente, en $4^{\circ}$ año la instrucción formal en aritmética tiene como meta que la población infantil pueda reconocer la estrategia menos costosa y sintética para la resolución de las operaciones (Ministerio de Educación, Ciencia y Tecnología de Argentina, 2011b). De este modo, la inteligencia fluida contribuiría al cálculo y aprendizaje de la división, lo cual permitiría la abstracción de las relaciones numéricas implicadas en los problemas, y la consecuente selección del procedimiento de cálculo menos costoso para su solución.

Por otro lado, en función de lo señalado en estudios previos (Siegler y Pyke, 2013), esperábamos hallar una relación de la capacidad de inhibición con el desempeño en división. No obstante, no observamos diferencias entre estudiantes con distinto nivel de desempeño en división en su capacidad de inhibición. Diversos estudios indicaron que el control cognitivo exigido en distintas estrategias de resolución de operaciones aritméticas varía (ver Geary et al., 2008 para una revisión), por lo que es posible que la relación de la inhibición con el cálculo de la división difiera conforme al tipo de estrategia de solución implementada por el estudiantado. Lamentablemente, ni en nuestro estudio ni en el de Siegler y Pyke (2013) se controló el efecto del tipo de estrategia implementada por los niños en la relación de procesos de control cognitivo con el cálculo de la división. Esta limitación deberá ser corregida en futuros trabajos.

En síntesis, este trabajo contribuye a la literatura al indicar relaciones específicas entre el nivel de desempeño en división y la capacidad de MT e inteligencia fluida. Estos resultados presentan ciertas implicaciones a nivel de las prácticas educativas. En primer lugar, sugieren que adecuar la exigencia de retención y procesamiento de la información a los recursos de MT del estudiantado los estudiantes, facilitaría el cálculo de la división y su dominio. Los recursos de retención y procesamiento de la información en la MT son limitados, por lo que su apropiada demanda permitiría el logro del cálculo divisorio, su comprensión y aprendizaje. La carga de MT variará en diferentes problemas según la estrategia de cálculo que empleen los y las estudiantes, por lo que resultaría apropiado 
anticipar las posibles estrategias de solución que pueden establecer al seleccionar problemas para su enseñanza.

Las estrategias de cálculo se establecen en función del conocimiento previo de los estudiantes, de ahí que resulte necesario considerar también dicho conocimiento en la selección de los problemas, y trabajar previamente para la consolidación de aquellos que permitan reducir la carga de MT. Por ejemplo, diferentes autores indicaron que la fluidez (precisión y velocidad) en la realización de operaciones aritméticas simples (e.g., 5-3=2) facilita la correcta ejecución de cálculos que involucran relaciones numéricas de mayor complejidad (ver Geary et al., 2008 para revisión). Esto se debe a que la recuperación automática de los resultados de cálculos simples, permite destinar los recursos limitados de procesamiento de la MT al control y monitoreo en la realización de operaciones más complejas (e.g., operaciones de división complejas). En función de lo anterior, el trabajo previo enfocado al logro de la fluidez en la recuperación de los resultados de operaciones simples facilitará el cálculo de divisiones de mayor complejidad.

Por otro lado, considerando las diferencias en inteligencia fluida entre estudiantes con diverso desempeño en división, sería conveniente utilizar durante su enseñanza situaciones problemáticas en las que la complejidad de las relaciones numéricas a identificar y abstraer se corresponda con la capacidad de inteligencia fluida de los mismos. Además, la explicitación de tales relaciones por parte del docente resultaría central para facilitar el aprendizaje de niños con menor capacidad de inteligencia fluida. Conforme a lo señalado por Broitman e Itzcovich (2001), la experiencia en la resolución de diferentes situaciones problemáticas de equivalente complejidad promovería el reconocimiento de las relaciones numéricas implicadas en los problemas, la elaboración de estrategias válidas de solución y su transferencia para la resolución de nuevos problemas. Una vez consolidadas ciertas nociones matemáticas y estrategias, podría incrementarse la complejidad de las relaciones implicadas en los problemas y avanzar en la enseñanza de este conocimiento.

\section{Referencias}

Adams, Julia y Weakliem, David. (2011). August B. Hollingshead's "Four Factor Index of Social Status": From Unpublished Paper to Citation Classic. Yale Journal of Sociology, 8, 11-20. Recuperado de https://www.academia.edu/2798550/August B. Hollingshead s Four Factor Index of Social Status From Unpublished Paper to Citation Classic?auto=download 
Ancker, Jessica S. y Kaufman, David. (2007). Rethinking health numeracy: a multidisciplinary literature review. Journal of the American Medical Informatics Association, 14(6), 71321. doi: http://dx.doi.org/10.1197/jamia.M2464

Andrés, María Laura, Castañeiras, Claudia, y Richaud de Minzi, María Cristina (2014). Relaciones entre la personalidad y el bienestar emocional en niños. El rol de la regulación emocional. Cuadernos de Neuropsicología, 8(2), 217-241. Recuperado de https://dialnet.unirioja.es/servlet/articulo?codigo $=4940727$

Andrés, María Laura, Richaud de Minzi, María Cristina, Castañeiras, Claudia, Canet-Juric, Lorena, y Rodríguez-Carvajal, Raquel. (2016). Neuroticism and Depression in Children: The Role of Cognitive Emotion Regulation Strategies. The Journal of Genetic Psychology, 177(2), 55-71. doi: http://dx.doi.org/10.1080/00221325.2016.1148659

Anghileri, Julia. (2001). Development of division strategies for Year 5 pupils in ten English schools. British Educational Research Journal, 27(1), 85-103. Recuperado de http://onlinelibrary.wiley.com/doi/10.1080/01411920125581/epdf

Anghileri, Julia, Beishuizen, Meindert y Van Putten, Kees. (2002). From informal strategies to structured procedures: Mind the gap! Educational Studies in Mathematics, 49(2) 149_ 170. doi: http://dx.doi.org/10.1023/A:1016273328213

Aragón, Estíbaliz, Delgado, Cándida, Aguilar, Manuel, Araujo, Antonio y Navarro, José. (2013). Estudio de la influencia de la inteligencia y el género en la evaluación matemática temprana. European Journal of Education and Psychology, 6(1), 5-18. Recuperado de https://formacionasunivep.com/ejep/index.php/journal/article/view/90

Baddeley, Alan D., y Hitch, Graham J. (1974). Working memory. En Gordon Bower (Ed.) The Psychology of Learning and Motivation: Advances in Research and Theory (pp. 47-89). New York: Academic.

Baddeley, Alan. (2012). Working memory: theories, models, and controversies. Annual Review of Psychology, 63, 1-29. Recuperado de http://www.annualreviews.org/doi/abs/10.1146/annurev-psych-120710-100422

Bellon, Elien, Fias, Wim y De Smedt, Bert. (2016) Are Individual Differences in Arithmetic Fact Retrieval in Children Related to Inhibition? Frontiers in Psychology, 7, 1-11. Recuperado de https://biblio.ugent.be/publication/7258233

Boero, Paolo, Ferrari, Pier Luigi, y Ferrero, Enrica (1989). Division problems: Meanings and procedures in the transition to a written algorithm. For the Learning of Mathematics, 9(3), 17-25. Recuperado de https://www.jstor.org/stable/40248158?seq=1\#page scan tab contents

Broitman, Claudia e Itzcovich, Horacio. (2001). Orientaciones didácticas para la enseñanza de la división en los tres ciclos de la EGB. Recuperado de http://servicios2.abc.gov.ar/lainstitucion/sistemaeducativo/educprimaria/areascurricular es/matematica/division.pdf 
Broitman, Claudia, Escobar, Mónica, Etchemendy, Mercedes, Novembre, Andrea, y Sancha, Inés. (2011). Estudiar Matemática en 4ํ․ Buenos Aires: Santillana.

Bull, Rebecca, Espy, Kimberly A., y Wiebe, Sandra A. (2008). Short-Term Memory, Working Memory, and Executive Functioning in Preschoolers: Longitudinal Predictors of Mathematical Achievement at Age 7 Years. Developmental Neuropsychology, 33(3), 205-228. doi: http://dx.doi.org/10.1080/87565640801982312

Canet-Juric, Lorena, Introzzi, Isabel, y Burin, Débora. (2015). Desarrollo de la Capacidad de Memoria de Trabajo Efectos de Interferencia Inter e Intra Dominio en Niños de Edad Escolar. Revista Argentina de Ciencias del Comportamiento, 7(1), 26-37. Recuperado de http://www.redalyc.org/pdf/3334/333439929004.pdf

Cattell, Raymond. B. (1987). Intelligence: Its Structure, Growth and Action. Amsterdam: North-Holland.

Cayssials, Alicia. (1993). Test de Matrices Progresivas. Manual (adaptación). Argentina, Buenos Aires: Paidós.

Censabella, Sandrine, y Noël, Marie Pascal (2005). The inhibition of exogenous distracting information in children with learning disabilities. Journal of Learning Disabilities, 38(5), 400-410. Recuperado http://journals.sagepub.com/doi/pdf/10.1177/00222194050380050301

Censabella, Sandrine, y Noël, Marie Pascal. (2007). The inhibition capacities of children with mathematical disabilities. Child Neuropsychology, 14(1), 1-20. doi: http://dx.doi.org/10.1080/09297040601052318

Cragg, Lucy y Gilmore, Camila. (2014). Skills underlying mathematics: The role of executive function in the development of mathematics proficiency. Trends in Neuroscience and Education, 3(2), 63-68. doi: http://dx.doi.org/10.1016/i.tine.2013.12.001

D'Amico, Antonella, y Passolunghi, Maria Chiara (2009). Naming speed and effortful and automatic inhibition in children with arithmetic learning disabilities. Learning and Individual Differences, 19(2), 170-180. doi: http://dx.doi.org/10.1016/i.lindif.2009.01.001

Departamento de Evaluación de la Calidad Educativa (Diniece). (2013). Operativo Nacional de Evaluación ONE 2013. Informe Nacional de Resultados muestra de $3^{\circ}$ y 6o año Educación Primaria. Recuperado de http://portales.educacion.gov.ar/diniece/wpcontent/blogs.dir/37/files/2015/07/INFORME-DE-RESULTADOS-PRIMARIA-ONE2013.pdf

Diamond, Adel. (2013). Executive functions. Annual Review of Psychology, 64, 135-168. Recuperado de http://www.annualreviews.org/doi/full/10.1146/annurev-psych-113011143750

Every Child a Chance Trust. (2009). The long-term costs of numeracy difficulties (2 ${ }^{\text {nd }}$ ed.). Recuperado de https://readingrecovery.org/wpcontent/uploads/2016/12/long term costs of literacy difficulties 2nd edition 2009.pdf 
Fagginger Auer, Marije F. (2016) Solving multiplication and division problems: latent variable modeling of students' solution strategies and performance. (Tesis doctoral, Social and Behavioural Sciences Leiden). Recuperado de https://openaccess.leidenuniv.nl/handle/1887/40117

Ferrer, Emilio y McArdle, Jhon J. (2004). An experimental analysis of dynamic hypotheses about cognitive abilities and achievement from childhood to early adulthood. Developmental Psychology, 40(6), 935-952. doi: http://dx.doi.org/10.1037/0012$\underline{1649.40 .6 .935}$

Ferrer, Emilio, O' Hare, Elizabeth D., y Bunge, Silvia A. (2009). Fluid reasoning and the developing brain. Frontiers in neuroscience, 3(1), 46-51. Recuperado de https://www.ncbi.nlm.nih.gov/pmc/articles/PMC2858618/

Flotts, M. Paulina, Manzi, Jorge, Jiménez, Daniela, Abarzúa, Andrea, Cayumán, Carlos y García, María José. (2015). Informe de resultados Tercer Estudio Comparativo y Explicativo (TERCE) del Laboratorio Latinoamericano de Evaluación de la Calidad de la Educación. Logros de aprendizaje. Santiago: OREALC/UNESCO. Recuperado de http://unesdoc.unesco.org/images/0024/002435/243532S.pdf

Fuchs, Lynn, Fuchs, Douglas, Compton, Donald, Powell, Sarah R., Seethaler, Pamela M., Capizzi, Andrea M., y Fletcher Jack M. (2006). The cognitive correlates of third-grade skill in arithmetic, algorithmic computation, and arithmetic word problems. Journal of Educational Psychology, 98(1), 29-43. doi: http://dx.doi.org/10.1037/0022-0663.98.1.29

Geary, David, Boykin, Wade A., Embretson, Susan, Reyna, Valerie, Siegler, Robert, Berch, Daniel B. y Graban, Jeniffer. (2008). Report of the task group on learning processes. In U.S. Department of Education (Ed.), The final report of the National Mathematics Advisory Panel (pp. 4-1 - 4-221, Capítulo 4). Washington, DC: U.S. Department of Education. Recuperado de http://www.ed.gov/about/bdscomm/list/mathpanel/report/learning-processes.pdf

Gilmore, Camila, Attridge, Nina, Clayton, Sarah, Cragg, Lucy, Johnson, Samantha, Marlow, Neil, y Inglis, Mathew. (2013). Individual differences in inhibitory control, not non-verbal number acuity, correlate with mathematics achievement. PloS one, 8(6), e67374. doi: http://dx.doi.org/10.1371/journal.pone.0067374

Grinyer, John. (2005). Literacy, numeracy and the labour market. London: DfES

Hansen, Nicole, Jordan, Nancy C., Fernandez, Edmun, Siegler, Robert S., Fuchs, Lynn, Gersten, Rusell y Micklos, Deborah. (2015). General and math-specific predictors of sixth-graders' knowledge of fractions. Cognitive Development, 35, 34-49. doi: http://dx.doi.org/10.1016/j.cogdev.2015.02.001

Hanushek, Eric A. y Woessmann, Ludger. (2010). The High Cost of Low Educational Performance: The Long-Run Economic Impact of Improving PISA Outcomes. OECD Publishing. Recuperado de https://www.oecd.org/pisa/44417824.pdf 
Hecht, Steven A. y Vagi, Kevin J. (2010). Sources of group and individual differences in emerging fraction skills. Journal of Educational Psychology, 102(4), 843-859. doi: http://dx.doi.org/10.1037/a0019824

Hickendorff, Marian, van Putten, Cornelius M., Verhelst, Norman D., y Heiser, Willem. J. (2010). Individual differences in strategy use on division problems: Mental versus written computation. Journal of Educational Psychology, 102(2), 438. doi: http://dx.doi.org/10.1037/a0018177

Hollingshead, August. B. (2011). Four Factor Index of Social Status. Yale Journal of Sociology, 8, 21-52.

Hudson, Melissa E., Browder, Diana M. y Wood, Leah A. (2013). Review of experimental research on academic learning by students with moderate and severe intellectual disability in general education. Research and Practice for Persons with Severe Disabilities, 38(1), 17-29. Recuperado de http://journals.sagepub.com/doi/abs/10.2511/027494813807046926

Introzzi, Isabel, Andrés, María Laura, Canet-Juric, Lorena, Stelzer, Florencia y Richard's, María. (2016). The relationship between the rumination style and perceptual, cognitive and behavioral inhibition. Psychology y Neuroscience, 9(4), 444-456. doi: http://dx.doi.org/10.1037/pne0000068

Jarvis, Helen y Gathercole, Susan. (2003). Verbal and non-verbal working memory and achievements on National Curriculum tests at 11 and 14 years of age. Educational and Child Psychology, 20(3), 123-140.

Jordan, Nancy C., Kaplan, David, Ramineni, Chaitanya, y Locuniak, Maria N. (2009). Early math matters: kindergarten number competence and later mathematics outcomes. Developmental Psychology, 45(3), 850. Recuperado de https://www.ncbi.nlm.nih.gov/pmc/articles/PMC2782699/

Kratzer, Richard O. y Willoughby, Stephen. (1973). A comparison of initially teaching division employing the distributive and Greenwood algorithms with the aid of a manipulative material. Journal for Research in Mathematics Education, 4(4), 197-204. doi: http://dx.doi.org/10.2307/748599

LeFevre, Jo-Anne y Morris, Jason. (1999). More on the relation between division and multiplication in simple arithmetic: Evidence for mediation of division solutions via multiplication. Memory y Cognition, 27(5), 803-812. doi: http://dx.doi.org/10.3758/BF03198533

Mayes Dickerson, Susan y Calhoun, Susan L. (2006). Frequency of reading, math, and writing disabilities in children with clinical disorders. Learning and Individual Differences, 16(2), 145-157. doi: https://doi.org/10.1016/j.lindif.2005.07.004

McArdle, John. J., Ferrer-Caja, Emilio, Hamagami, Fumiaki, y Woodcock, Richard W. (2002). Comparative longitudinal structural analysis of growth and decline of multiple intellectual abilities over the lifespan. Developmental Psychology, 38(1), 113-142. doi: http://dx.doi.org/10.1037//0012-1649.38.1.115 
McArdle, John J., Hamagami, Fumiaki, Meredith, William y Bradway, Kathering P. (2000). Modeling the dynamic hypotheses of Gf-Gc theory using longitudinal life-span data. Learning and Individual Differences, 12(1), 53-79. doi: http://dx.doi.org/10.1016/S1041$\underline{6080}(00) 00036-4$

McCormick, Marie C., Litt, Jonathan S., Smith, Vincent C. y Zupancic, John. A. (2011). Prematurity: an overview and public health implications. Annual review of public health, 32, 367-379. doi: https://doi.org/10.1146/annurev-publhealth-090810-182459

Meyer, Megahn L., Salimpoor, Valorie N., Wu, Sarah. S., Geary, David C., y Menon, Vinod. (2010). Differential contribution of specific working memory components to mathematics achievement in 2nd and 3rd graders. Learning and Individual Differences, 20(2), 101109. doi: https://doi.org/10.1016/i.lindif.2009.08.004

Ministerio de Educación, Ciencia y Tecnología. (2007a). Cuadernos para el aula, matemática 2. Recuperado de http://www.me.gov.ar/curriform/nap/2do matem.pdf

Ministerio de Educación, Ciencia y Tecnología de Argentina. (2007b). Cuadernos para el aula, matemática $3 . \quad$ Recuperado de http://www.me.gov.ar/curriform/nap/3ero matema.pdf

Ministerio de Educación, Ciencia y Tecnología de Argentina. (2007c). Cuadernos para el aula, matemática 4.4 Recuperado de http://www.me.gov.ar/curriform/nap/matematica4 final.pdf

Ministerio de Educación, Ciencia y Tecnología de Argentina. (2011a). Núcleos de Aprendizajes Prioritarios Primer Ciclo EGB / Nivel Primario. Recuperado de http://www.me.gov.ar/curriform/publica/nap/nap-egb-primario.pdf

Ministerio de Educación, Ciencia y Tecnología de Argentina. (2011b). Núcleos de Aprendizajes Prioritarios Segundo Ciclo EGB / Nivel Primario. Recuperado de http://www.me.gov.ar/curriform/publica/nap/nap egb2.pdf

Namkung, Jessica M. y Fuchs, Lynn S. (2015). Cognitive predictors of calculations and number line estimation with whole numbers and fractions among at-risk students. Journal of Educational Psychology, 108(2), 214-228. doi: http://dx.doi.org/10.1037/edu0000055

Navarro, José I., Aguilar, Manuel, Alcalde, Concepción, Ruiz, Gonzalo, Marchena, Esperanza, y Menacho, Inmaculada. (2011). Inhibitory processes, working memory, phonological awareness, naming speed, and early arithmetic achievement. The Spanish Journal of Psychology, 14(02), 580-588. doi: http://dx.doi.org/10.5209/rev SJOP.2011.v14.n2.6

Organización para la Cooperación y el Desarrollo Económicos (OCDE). (2014). Resultados de PISA 2012 en Foco. Lo que los alumnos saben a los 15 años de edad y lo que pueden hacer con lo que saben. Recuperado de https://www.oecd.org/pisa/keyfindings/PISA2012 Overview ESP-FINAL.pdf 
Paglin, Morton y Rufolo, Antony M. (1990). Heterogeneous human capital, occupational choice, and male-female earnings differences. Journal of Labor Economics, 8(1), 123144.

Parsons, Shamanta y Bynner, John. (2005). Does numeracy matter more? National Research and Development Centre for Adult Literacy and Numeracy (Research Report). London: Institute of Education. Recuperado de http://eprints.ioe.ac.uk/4758/1/parsons2006does.pdf

Pascual, Liliana, Galperín, Celia Z. y Bornstein, Marc H. (1993). La medición del nivel socioeconómico y la psicología evolutiva: El caso Argentino. Revista Interamericana de Psicologia/Interamerican Journal of Psychology, 27(1), 59-74. Recuperado de https://journal.sipsych.org/index.php/IJP/article/view/786/680

Peng, Peng, Namkung, Jessica M., Fuchs, Douglas, Fuchs, Lynn S., Patton, Samuel, Yen, Loulee, ... y Hamlett, Carol. (2016). A longitudinal study on predictors of early calculation development among young children at risk for learning difficulties. Journal of Experimental Child Psychology, 152, 221-241. doi: https://doi.org/10.1016/i.jecp.2016.07.017

Piccinini, Cesar A., Tudge, Jonathan, Marín, Ángela H., Bitencourt-Frizzo, Giana, y CássiaSobreira Lopes, Rita de. (2010). The impact of socio-demographic variables, social support and child sex on mother-infant and father-infant interaction. Interamerican Journal of Psychology, 44(2), 382-391. Recuperado de http://www.redalyc.org/pdf/284/28420641019.pdf

Raghubar, Kimberly P., Barnes, Marcia A. y Hecht, Steven A. (2010). Working memory and mathematics: A review of developmental, individual difference, and cognitive approaches. Learning and Individual Differences, 20(2), 110-122.

Raven, J.C. (1989): Test de Matrices Progresivas para la medida de la capacidad intelectual, de sujetos de 4 a 11 años. Buenos Aires: Paidós.

Richard's, María, Introzzi, Isabel, Zamora, Eliana y Vernucci, Santiago. (2017). Analysis of Internal and External Validity Criteria for a Computerized Visual Search Task. A pilot study. Applied Neuropsychology: Child, 6(2), 110-119. doi: http://dx.doi.org/10.1080/21622965.2015.1083433

Richard's, María, Vernucci, Santiago, Zamora, Eliana, Canet-Juric, Lorena, Introzzi, Isabel, y Guardia, Joan (2017). Contribuciones empíricas para la validez de grupos contrastados de la Batería de Tareas de Autorregulación Cognitiva (TAC). Interdisciplinaria, 34(1), 173-192.

Rivera-Batiz, Francisco L. (1992). Quantitative literacy and the likelihood of employment among young adults in the United States. Journal of Human Resources, 27(2), 313328. doi: http://dx.doi.org/10.2307/145737

Robinson, Katherine M., Arbuthnott, Katherine D., Rose, Danica, McCarron, Michelle C., Globa, Carin A., y Phonexay, Sylvia D. (2006). Stability and change in children's division strategies. Journal of Experimental Child Psychology, 93(3), 224-238. 
Rose, Heather y Betts, Julian R. (2004). The effect of high school courses on earnings. Review of Economics and Statistics, 86(2), 497-513. doi: http://dx.doi.org/10.1162/003465304323031076

Sautú, Roberto. (1989). Teoría y técnica en la medición del status ocupacional: Escalas objetivas de Prestigio. (Documento de trabajo). Buenos Aires: Universidad de Buenos Aires. Instituto de Ciencias Sociales.

Siegler, Robert S., Duncan, Greg J., Davis-Kean, Pamela E., Duckworth, Kathryn, Claessens Amy, Engel Mimi, Susperreguy Maria Ines y Chen Meichu. (2012). Early predictors of high school mathematics achievement. Psychological Science, 23(7), 691- 697. Recuperado de http://journals.sagepub.com/doi/pdf/10.1177/0956797612440101

Siegler, Robert S. y Pyke, Aryn A. (2013). Developmental and individual differences in understanding of fractions. Developmental Psychology, 49(10), 1994-2004. doi: http://dx.doi.org/10.1037/a0031200

Stock, Pieter, Desoete, Annemie y Roeyers, Hebert. (2009). Predicting arithmetic abilities: The role of preparatory arithmetic markers and intelligence. Journal of Psychoeducational Assessment, 27(3), 237-251. Recuperado de https://biblio.ugent.be/publication/790966

Treisman, Anne y Gelade, Garry. (1980). A feature integration theory of attention. Cognitive Psychology, 12(1), 97-136.

Van Der Ven, Sanne H., Van Der Maas, Hann L., Straatemeier, Marthe y Jansen, Brenda R. (2013). Visuospatial working memory and mathematical ability at different ages throughout primary school. Learning and Individual Differences, 27, 182-192. doi: http://dx.doi.org/10.1016/i.lindif.2013.09.003

van der Sluis, Sophie, de Jong, Peter F., y van der Leij, Aryan. (2004). Inhibition and shifting in children with learning deficits in arithmetic and reading. Journal of Experimental Child Psychology, 87(3), 239-266. doi: http://dx.doi.org/10.1016/i.jecp.2003.12.002

Van de Weijer-Bergsma, Eva, Kroesbergen, Evelyn H. y Van Luit, Johannes E. (2015). Verbal and visual-spatial working memory and mathematical ability in different domains throughout primary school. Memory y cognition, 43(3), 367-378. doi: http://dx.doi.org/10.3758/s13421-014-0480-4

Van Putten, Cornelius M., Van den Brom-Snijders, Petra A. y Beishuizen, Meindert. (2005). Progressive mathematization of long division strategies in Dutch primary schools. Journal for Research in Mathematics Education, 36(1), 44-73. Recuperado de https://www.jstor.org/stable/30034920?seq=1\#page scan tab contents

Vukovic, Rose K., Fuchs, Lynn S., Geary, David C., Jordan, Nancy C., Gersten, Russell, y Siegler, Robert S. (2014). Sources of individual differences in children's understanding of fractions. Child Development, 85(4), 1461-1476. doi: http://dx.doi.org/10.1111/cdev.12218 